\title{
Coherent kilo-electron-volt backscattering from plasma-wave boosted relativistic electron mirrors
}

\author{
F. Y. Li, ${ }^{1}$ Z. M. Sheng, ${ }^{1,2, a)}$ M. Chen, ${ }^{1, b)}$ H. C. Wu, ${ }^{3}$ Y. Liu, ${ }^{1}$ J. Meyer-ter-Vehn, ${ }^{4}$ W. B. Mori, ${ }^{5}$ \\ and J. Zhang ${ }^{1}$ \\ ${ }^{1}$ Key Laboratory for Laser Plasmas (Ministry of Education) and Department of Physics and Astronomy, \\ Shanghai Jiao Tong University, Shanghai 200240, China \\ ${ }^{2}$ SUPA, Department of Physics, University of Strathclyde, Glasgow G4 ONG, United Kingdom \\ ${ }^{3}$ Institute for Fusion Theory and Simulation, Zhejiang University, Hangzhou 310027, China \\ ${ }^{4}$ Max-Planck-Institut für Quantenoptik, D-85748 Garching, Germany \\ ${ }^{5}$ University of California, Los Angeles, California 90095-1547, USA
}

(Received 20 August 2014; accepted 11 October 2014; published online 20 October 2014)

\begin{abstract}
A different parameter regime of laser wakefield acceleration driven by sub-petawatt femtosecond lasers is proposed, which enables the generation of relativistic electron mirrors further accelerated by the plasma wave. Integrated particle-in-cell simulation, including both the mirror formation and Thomson scattering, demonstrates that efficient coherent backscattering up to keV photon energy can be obtained with moderate driving laser intensities and high density gas targets. (C) 2014 AIP Publishing LLC. [http://dx.doi.org/10.1063/1.4899136]
\end{abstract}

Recently, tremendous interest for $\mathrm{x}$-ray generation has been drawn to intense laser-matter interactions. They promise ultrashort ultracompact $\mathrm{x}$-ray sources compared with large conventional facilities such as $\mathrm{x}$-ray free electron lasers. ${ }^{1}$ Various schemes have so far been developed including high harmonic generation from either gas $^{2,3}$ or solid targets ${ }^{4}$ laser-driven $K \alpha$ sources, ${ }^{5,6}$ plasma acceleration based betatron radiation, ${ }^{7}$ etc. Among these schemes, a simple and efficient approach is based on laser-driven relativistic electron mirrors. They may compress femtosecond probe pulses to attoseconds and boost photon energy by Doppler shifts of $\Gamma=4 \gamma_{x}^{2}$, where $\gamma_{x}=\left(1-v_{x}^{2} / c^{2}\right)^{-1 / 2}$ is the relativistic Lorentz factor related to the mirror's normal velocity $v_{x}$. Laser-driven plasmas provide a rich source of such mirrors ${ }^{8}$ and some involving interaction with solids ${ }^{4,9-12}$ are dense enough for coherent backscattering.

In underdense plasma, density crests of strongly driven plasma waves (or when close to wave breaking) develop converging spikes and have also been suggested as relativistic flying mirrors. ${ }^{13-15}$ This regime is of particular interest for experiments because high repetition rates are possible with gas targets as well as using less challenge conditions compared with the solid schemes which demand extremely high laser contrast. However, there remain a couple of issues when using the flying mirror for coherent backscattering up to $\mathrm{keV}$ photon energy. They are mainly due to the limitation for the Doppler factor determined by the phase velocity of the plasma wave $\left(v_{p}\right)$, i.e., $\Gamma \leq 4 \gamma_{p}^{2}=4 /\left(1-v_{p}^{2} / c^{2}\right)$ $\simeq 4 n_{c} / n_{0} ; n_{0}$ and $n_{c}$ are the ambient electron density and the critical density, respectively. ${ }^{15,16}$ The existing experiments ${ }^{17-19}$ have suggested limited $\Gamma<100$ for $n_{0}$ at a few $10^{19} \mathrm{~cm}^{-3}$. Reducing $n_{0}$ not only can give larger $\Gamma$ but also requires stronger driver to approach the wavebreaking limit. ${ }^{20}$ Meanwhile, it is preferred that a broad laser focal spot, e.g., $\sigma_{0}>\sqrt{a_{0}} \lambda_{p} / \pi$, can be used to drive the wake in the quasi-one-dimensional (quasi-1D) regime $e^{21,22}$ so that it

\footnotetext{
a) zhengming.sheng@strath.ac.uk and zmsheng@sjtu.edu.cn

b) minchen@sjtu.edu.cn
}

can provide a flat mirror plane for collimated backscattering. Here, $\lambda_{p}$ is the plasma wavelength and $a_{0}=8.5 \times$ $10^{-19} \lambda_{0}[\mu \mathrm{m}] \sqrt{\mathrm{I}_{0}\left[\mathrm{~W} / \mathrm{cm}^{2}\right]}$ is the normalized laser amplitude with $\lambda_{0}$ being the wavelength and $I_{0}$ being the peak intensity. Tremendous incident peak power, $P_{0} \propto a_{0}^{2} \sigma_{0}^{2} \propto n_{0}^{-4}$, is then required at low $n_{0}$ for high $\Gamma$ factors. More critically, the exact breaking point, crucial for efficient backscattering, is actually hard to achieve in quasi-1D thermal plasmas. ${ }^{23}$ Instead, wave breaking often occurs as the laser pulse evolves significantly (including self-modulation and self-focusing) and drives the wake in the bubble regime ${ }^{24,25}$ with an evidence of generating energetic electron beams. ${ }^{19}$ As a result, backscattering off such near-spherical bubble shell will lead to extremely large radiation divergence.

The aim of this letter is to report a scenario that can overcome the above shortages and result in coherent Thomson backscattering up to $\mathrm{keV}$ photon energy using reasonable driver conditions. The key point is to break the constraint set by the wave's phase velocity $v_{p}$. The way to achieve this is to drive the wake even harder and let the mirror be properly injected into the plasma wave. Consequently, the Doppler factor of the mirror can be boosted by wakefield acceleration, ${ }^{26}$ not limited by $v_{p}$ any more. As we shall see, the only limitation now turns out to be $\gamma_{x}$ rising above some threshold, at which the scattered light degrades into incoherent pulses. With this scenario, low density $n_{0}$ is not necessarily used for high $\Gamma$ factors. Instead, high density gas targets can be employed which, as we have argued, will reduce the peak laser power required.

It is clear that synchronous injection of the density wave spike is desired so that the mirror spike can be accelerated as a whole. Normally, quasi-continuous injection occurs, which leads to narrow electron bunches of femtosecond duration. Such bunches have been used for incoherent backscattering. ${ }^{27-29}$ Here, to make the sharp injection possible, two steps are necessary. The first is to drive the wake close to breaking but without injection. Specifically, a density upramp can be used to suppress electron injection for the first few wake wave periods trailing after the driving laser. ${ }^{30,31}$ 
For the ramp of high enough gradients, sequential contraction of the plasma wavelength (along the laser direction) dominates so that the wave can travel at superluminal speeds. As a result, at high nonlinearities, the density crests can be stably compressed into spikes without premature injection. This free of injection eventually terminates as the laser pulse propagates through the ramp to the following uniform region where the wave's phase speed becomes subluminal. It refers to the second step as the sharp injection. As the phase speed falls below the light speed abruptly at the density transition region, a major part of the tightly compressed density crest is injected as a whole. Details of the controlled injection are described in a previous publication. ${ }^{30}$

This sharp injection actually works over a wide range of plasma densities and for given density the laser amplitude only has to meet some threshold. Here, in order to drive the boosted mirrors with reasonable laser conditions, we propose using high density gas plasmas $\left(\sim 10^{20} \mathrm{~cm}^{-3}\right)$ so that a laser focal spot of $10 \sim 20 \mu \mathrm{m}$ is sufficient for wake excitation in the quasi-1D regime. Notice that most experiments on wakefield acceleration are currently operated in the bubble regime with low densities $\left(10^{17} \sim 10^{18} \mathrm{~cm}^{-3}\right)$ for the generation of $\mathrm{GeV}$ beams. ${ }^{32,33}$ However, it is the essence of the new parameter regime that allows dense electron sheets to be trapped and the consequent generation of coherent keV backscattering instead of the incoherent sources normally obtained so far. ${ }^{7,27,28}$

Below we demonstrate coherent backscattering off the injected mirror via particle-in-cell (PIC) simulations. ${ }^{34}$ At first, 1D PIC simulations are used to illustrate the basic features. The interaction geometry is shown in Fig. 1(a). A 12.5-cycle driving laser, polarized along $z$ axis, propagates in the $+x$ direction with dimensionless amplitude $a_{0}=6$, corresponding to $I_{0}=7.7 \times 10^{19} \mathrm{~W} / \mathrm{cm}^{2}$ for $\lambda_{0}=0.8 \mu \mathrm{m}$. The plasma density is $n_{0}=7 \times 10^{19} \mathrm{~cm}^{-3}$ at $x \in[45,75] \mu \mathrm{m}$ with a $45 \mu \mathrm{m}$ long ramping front. The probe pulse, polarized along $y$ axis, propagates in the opposite direction and is appropriately delayed so that it meets the first density spike shortly after the sharp injection. The probe pulse with amplitude $a_{p r}=0.1$ has the same frequency as the driver $\left(\omega_{p r}=\omega_{0}=2 \pi c / \lambda_{0}\right)$ and takes a 12.5-cycle rectangle shape in time domain. In the simulation, 4000 cells $/ \mu \mathrm{m}$ are used to resolve the high-frequency backscattering. Anisotropic electron temperature is initialized, e.g., $10 \mathrm{~s} \mathrm{eV}$ transverse temperature and much lower for the longitudinal one. The low longitudinal temperature is acceptable in experiments as the electrons released from atoms by prepulse ionization are first dominant in transverse quiver motion at subpicosecond time scales.

The inset of Fig. 1(a) shows a closer look of the injected density spike. A remarkable feature is the very sharp front edge $^{35}$ which, as expanded in phase space $x-p_{y}$ [see the inset of Fig. 1(b)], shows a monoenergetic peak of $p_{x} \sim 10$ or $\gamma_{x} \simeq 10.5$. The evolution of this peak $\gamma_{x}$ around the injection instant is given in Fig. 1(b). It is seen that $\gamma_{x}$ is boosted by wakefield acceleration after $t=56 \mu \mathrm{m} / \mathrm{c}$. Before that it is kept at about 5.5, in consistent with the estimation from $\gamma_{p} \simeq \sqrt{n_{c} / n_{0}}=5$. Fig. 1(c), most importantly, presents the spectrum of the scattered pulse. It exhibits an ultrabroad bandwidth extending up to $k_{x} / k_{0} \simeq 1000$ or $1.5 \mathrm{keV}$ in
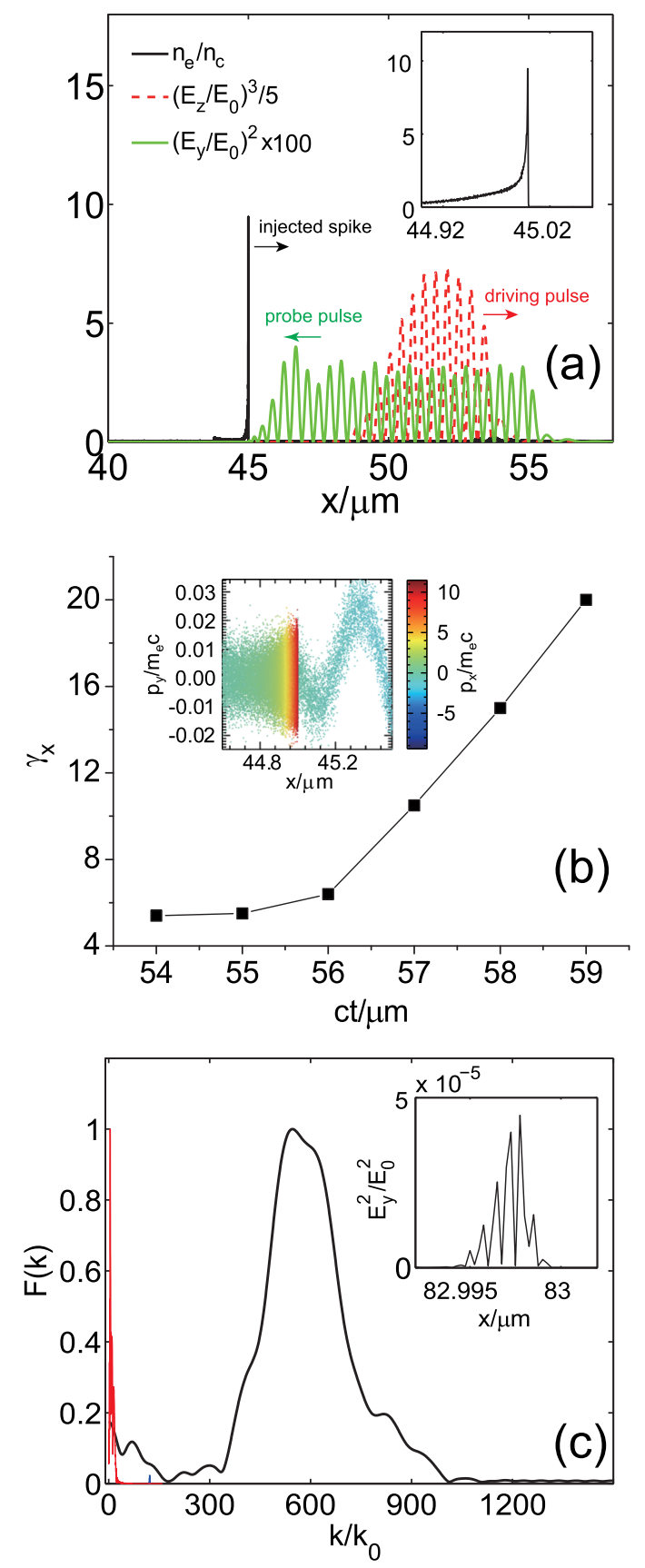

FIG. 1. Results of 1D PIC simulation. (a) Snapshot of electron density $n_{e}$, driving pulse $E_{z}^{2}$, and probe pulse $E_{y}^{2}$ at $t=57 \mu \mathrm{m} / \mathrm{c}$ shortly after the injection of the first density wave spike; inset shows a closer look of the spike with its phase space $x-p_{y}$ (colored according to $p_{x}$ ) in the inset of (b). (b) Evolution of peak $\gamma_{x}$ of the mirror spike around the time instant of injection; (c) spatial spectrum of the scattered pulse $E_{y}$ (corresponding intensity profile $E_{y}^{2}$ in the inset) from the injected spike. The red curve refers to the spectrum obtained for the driving laser of $a_{0}=2.5$ while keeping the other parameters fixed.

photon energy with $k_{0}=2 \pi / \lambda_{0}$. The corresponding spatial profile (see the inset therein) consists of only 3.5 cycles, rather than 12.5 cycles for the probe pulse. This selfcontraction effect ${ }^{36}$ is mainly ascribed to high enough $\gamma_{x}$, for which the coherent reflection condition $^{38}$ (i.e., $n_{e} \gg 10^{13} \gamma_{x}^{4} \mathrm{~cm}^{-3}$ ) is no longer satisfied and the backscattering becomes incoherent and orders of magnitude weaker in intensity. That means the scattered pulse can adjust itself as a few-cycle attosecond $\mathrm{x}$-ray pulse regardless of the incident 

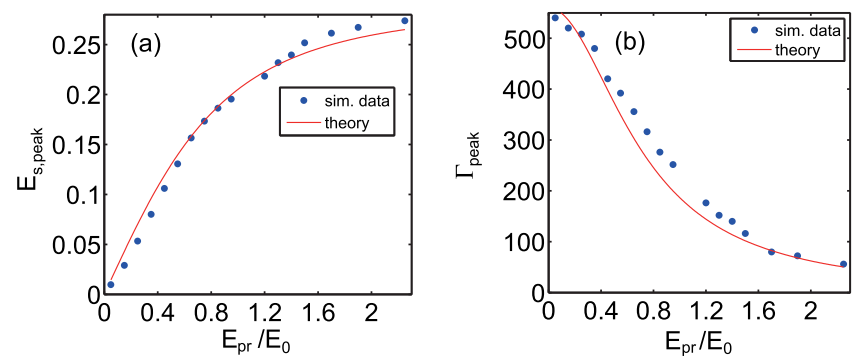

FIG. 2. (a) Peak scattering fields $E_{s, p e a k}$ and (b) frequency upshifting factor for the corresponding spectra peak $\Gamma_{\text {peak }}$ versus different probe laser amplitudes. Blue dots and red solid curves represent $1 \mathrm{D}$ simulation results and the nonlinear theory predications, respectively.

probe pulse length. Here, the coherent backscattering terminates at about $t=58.5 \mu \mathrm{m} / \mathrm{c}$ related to $\gamma_{x} \simeq 16$ or $\Gamma=1024$, which is in fair agreement with the maximal upshifted frequency visible. For comparison, the spectrum of reflection from the non-breaking density crest is also plotted in Fig. 1(c); only $\Gamma \simeq 16$ is obtained corresponding to $\gamma_{x} \simeq 2$.

The maximal amplitude of the scattered pulse [see the inset of Fig. 1(c)] is $E_{s, \text { peak }} / E_{0}=0.0063$ or $25 \mathrm{GV} / \mathrm{m}$, corresponding to a field reflectivity of $E_{s, p e a k} / E_{p r}=6.3 \%$, where $E_{0}=m_{e} \omega_{0} c / e$ is the normalizing field and $E_{p r}=a_{p r} E_{0}$ is the probe pulse field. The coherent reflectivity can also be estimated using the basic 1D model. ${ }^{37,38}$ For the sake of simplicity and also as indicated by the inset of Fig. 1(a), we use $n_{s}(x)=n_{s 0} \exp (x / d)$ (for $x \leq 0$ whereas zero) to approximate the density shape of the injected spike with $d$ being a characteristic thickness. The electric field reflectivity of the mirror is then derived as

$$
\eta=C \pi \frac{\Omega_{s 0}}{\omega_{p r}} \frac{d}{\Lambda_{s 0}} \frac{\gamma_{x}^{2}\left(1+\beta_{x}\right)}{\gamma},
$$

where $C=\left[1+16 d^{2} \pi^{2} \gamma_{x}^{4}\left(1+\beta_{x}\right)^{2} / \lambda_{p r}^{2}\right]^{-1 / 2}$ with $\lambda_{p r}$ being the probe laser wavelength, $\Omega_{s 0}=\sqrt{e^{2} n_{s 0} / \varepsilon_{0} m_{e}}$ is the plasma frequency associated with the maximal mirror density, and $\Lambda_{s 0}=2 \pi c / \Omega_{s 0}$. For the simulated parameters, $n_{s 0} \simeq 7.8 n_{c}, d \simeq 0.0025 \lambda_{0}$, and $\gamma_{x} \simeq 14$, the reflectivity amounts to $\eta \simeq 14.3 \%$, which is in reasonable agreement with the above observation. The infinitely sharp front of the spike used in the calculation may account for the slight overestimation by the theoretical model.

A series of simulations with different probe laser amplitudes (up to $E_{p r} / E_{0}>1$ ) is also conducted to study nonlinear backscattering off the boosted mirror. Figure 2 presents the maximal scattering fields $E_{s, p e a k}$ and the upshifting factors $\Gamma_{\text {peak }}$ for the corresponding spectra peak. For high-amplitude probe laser, its nonlinear ponderomotive force damps the mirror energy or the Doppler factor continuously as $E_{p r} / E_{0}$ increases, while $E_{s \text {,peak }}$ finally becomes saturated. These nonlinear features have been derived as $\Gamma_{\text {peak }} \simeq 4 \gamma_{x}^{2} /(1+$ $\left.E_{p r}^{2} / E_{0}^{2}\right)$ and $E_{s, p e a k}=E_{s a t}\left(E_{p r} / E_{0}\right)\left(1+E_{p r}^{2} / E_{0}^{2}\right)^{-1 / 2}$ with $E_{\text {sat }}$ being the saturated amplitude, ${ }^{36}$ and they show fair agreement with the simulation results.

To further explore the multidimensional effects of the boosted flying mirror concept, high resolution 2D PIC simulations (e.g., with the spatial mesh grid $1800 \times 100$ cells per square microns) are conducted. Notice that this resolution, though already high, is not yet sufficient to resolve the highest frequency that could be observed in the above 1D simulations which employed a higher resolution. To compensate this incapacity due to limited computational resources, highly nonlinear backscattering at $E_{p r} / E_{0}=1$ [the nonlinearity can reduce the effective $\Gamma$ factors as shown in Fig. 2(b)] is performed. The simulation parameters are similar to that for Fig. 1 except that a focal spot of $17 \mu \mathrm{m}$ (about 1.6 times the nonlinear plasma wavelength $\lambda_{n p}$ ) is used to drive the wake in the quasi-1D regime. Figure 3(a) shows the scattered pulse within a diameter of $10 \mu \mathrm{m}$. The scattered pulse amplitude is $E_{\text {scat }} / E_{0} \simeq 0.1$, corresponding to a peak intensity $\sim 10^{16} \mathrm{~W} / \mathrm{cm}^{2}$. The paraboloidal shape of the injected sheet arising from nonlinear plasma frequency shifts ${ }^{21}$ directly maps into the scattered pulse with a small curvature of $\sim 1 / 40 \mu \mathrm{m}^{-1}$, as shown in Fig. 3(a). More flat mirror planes for more collimated backscattering can be expected for a transversely super-Gaussian driving pulse.

Additional multidimensional effects come from the transverse momentum (e.g., $p_{y}$ in the $2 \mathrm{D}$ geometry) of the injected mirror, which holds even for non-breaking density wave crest and grows continuously during wakefield acceleration after injection. ${ }^{22}$ As documented in a number of papers, ${ }^{10,11,38}$ the transverse momenta may make $\Gamma=4 \gamma_{x}^{2}$

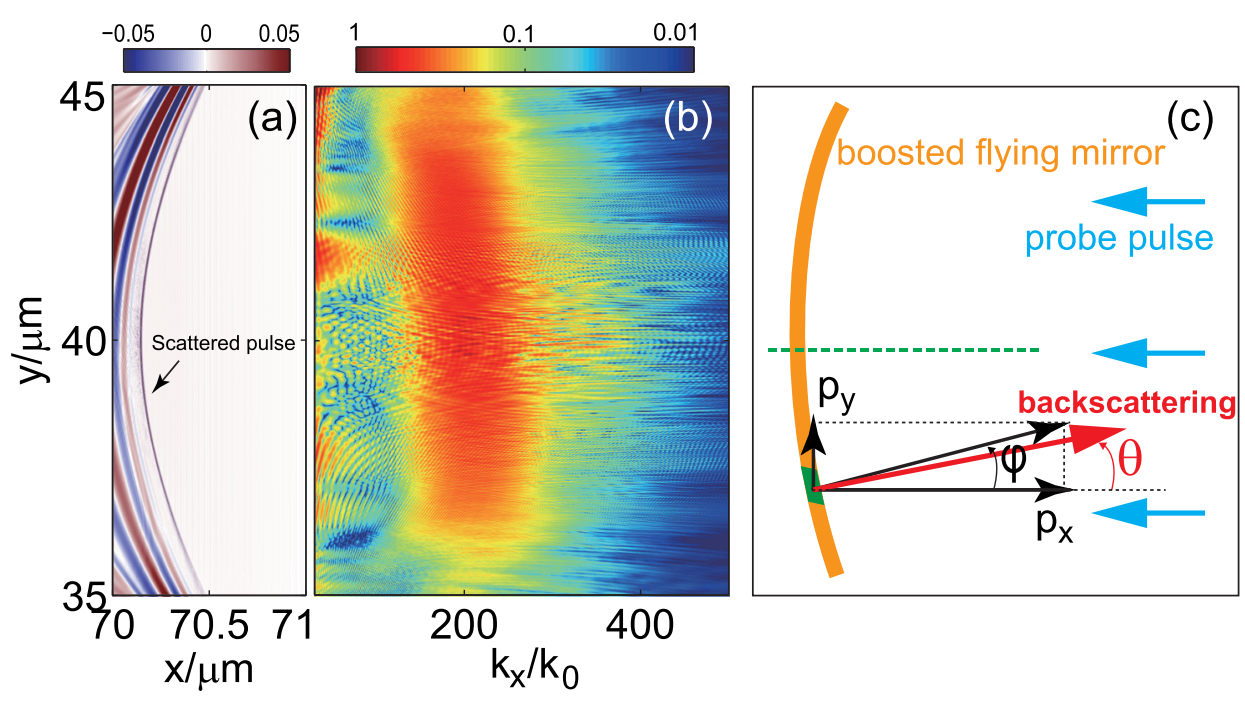

FIG. 3. Results of 2D PIC simulation. (a) Spatial profile of scattered pulse $E_{z} /$ $E_{0}$ (the probe laser is now polarized in $z$ direction while the driver in $y$ direction) and (b) corresponding spectrum in space $k_{x}-y$. (c) Schematic of backscattering off the boosted flying mirror, where $\varphi$ is the angle between $\vec{p}_{x}$ and the total momentum $\vec{p}$, and $\theta$ is the angle of the normal direction of the mirror segment relative to the $+x$ direction. 
significantly smaller than the full Doppler factor $4 \gamma^{2}$ with $\gamma=\gamma_{x}\left[1+\left(p_{y} / m_{e} c\right)^{2}\right]^{1 / 2}$. However, the scattered spectrum shown in space $k_{x}-y$ [see Fig. 3(b)] is almost uniform transversely. To account for this feature, we select a segment of the thin mirror [see the schematic in Fig. 3(c)] and analyze its behavior in backscattering. For coherent backscattering off a mirror with $\gamma \gg 1$, the scattered pulse is always directed close to the normal direction $(\vec{n})$ of the segment with angle $\theta$ relative to the $+x$ direction. ${ }^{38}$ For the present quasi-1D wave, $\theta$ is approximated by $\tan \theta=-d \lambda_{n p} / d y$ with $\lambda_{n p}$ being the plasma wavelength at each transverse position $y$. On the other hand, the segment electrons move at an angle defined as $\tan \varphi=p_{y} / p_{x}$. The momentum along the normal direction is then given by $p_{n}=p_{x} \cos \theta+p_{y} \sin \theta$. The near uniform spectrum shown above requires

$$
\Gamma_{n} \cos \theta \geq 4 \gamma_{x}^{2}
$$

where $\Gamma_{n}=4 /\left(1-p_{n}^{2} / m_{e}^{2} c^{2} \gamma^{2}\right)$ is the relativistic Doppler factor of this segment. With substitution of the above definitions, Eq. (2) is equivalent to $\left|d \lambda_{n p} / d r\right| \leq 2 p_{x} p_{y} /\left(p_{x}^{2}-p_{y}^{2} / 2\right.$ $-1 / 2$ ). This inequality sets an upper limit (about several times the angle of $\varphi$ ) for $\theta$ and can be readily met for the small curvature mirror driven in the quasi-1D regime. This effect can also be simply explained as that the tilt mirror surface deflects the coherent scattering close to the electron momenta direction, so that the full Doppler factors are almost recovered.

The uniform spectrum shows a peak at $k_{x} / k_{0} \sim 200$ which follows precisely the nonlinear 1D results of Fig. 2(b). This strongly suggests that the above 1D scaling for higher upshifting factors (e.g., up to $\mathrm{keV}$ level) also applies to high dimensions, though direct verifications are restricted by higher resolutions available. For the present case, the scattered pulse $(0.1 \mathrm{TW}$ peak power and $4 \mathrm{~nm}$ center wavelength) delivers over $10^{10}$ photons in 30 as. The energy conversion efficiency from the probe laser to the scattered $\mathrm{x}$ ray pulse is about a few $10^{-4}$. The high-flux coherent $\mathrm{x}$-ray pulses are acquired with a table-top facility that is typical for laser wakefield acceleration. ${ }^{27,28}$ They can be competitive with the large and expensive $\mathrm{x}$-ray free electron lasers in the peak power and also possess much shorter durations. In addition, the present scenario works in the quasi-1D regime so that it can be scaled up to larger driving focal spots delivered by multi-petawatt lasers.

In conclusion, we have proposed a different parameter regime of laser wakefield acceleration for coherent Thomson backscattering. This specific regime allows thin disk-like density wave crests to be compressed and trapped in the wake wave. They may serve as boosted mirrors with the Doppler factor no longer limited by the wave's phase velocity. Laser-like attosecond backscatterings up to $\mathrm{keV}$ photon energy have been derived using PIC simulations. Since high density gas targets are used, some hundred-terawatt lasers focused at about $10 \mu \mathrm{m}$ are sufficient to drive the boosted mirrors. These parameters are all within current technologies. The experimental implementation of the present scheme would also benefit from the advanced techniques developed for monitoring the backscattering at femtosecond time scales. ${ }^{12,19}$
Z.M.S. thanks the OSIRIS Consortium at UCLA and IST for providing access to OSIRIS 2.0 framework. This work was supported in part by the National Basic Research Program of China (Grant No. 2013CBA01504), the National Science Foundation of China (Grant Nos. 11121504, 11374210, and 11374209), and the MOST international collaboration project (Grant No. 2014DFG02330). Simulations were supported by Shanghai Supercomputer Center and the center for high performance computing at SJTU.

${ }^{1}$ B. W. McNeil and N. R. Thompson, Nat. Photonics 4, 814 (2010).

${ }^{2}$ P. Corkum and F. Krausz, Nat. Phys. 3, 381 (2007).

${ }^{3}$ F. Krausz and M. Ivanov, Rev. Mod. Phys. 81, 163 (2009).

${ }^{4}$ U. Teubner and P. Gibbon, Rev. Mod. Phys. 81, 445 (2009).

${ }^{5}$ L. M. Chen, M. Kando, M. Xu, Y. Li, J. Koga, M. Chen, H. Xu, X. Yuan, Q. Dong, Z. Sheng et al., Phys. Rev. Lett. 100, 045004 (2008).

${ }^{6}$ L. M. Chen, F. Liu, W. Wang, M. Kando, J. Mao, L. Zhang, J. Ma, Y. Li, S. Bulanov, T. Tajima et al., Phys. Rev. Lett. 104, 215004 (2010).

${ }^{7}$ S. Corde, K. T. Phuoc, G. Lambert, R. Fitour, V. Malka, A. Rousse, A. Beck, and E. Lefebvre, Rev. Mod. Phys. 85, 1 (2013).

${ }^{8}$ S. V. Bulanov, T. Z. Esirkepov, M. Kando, A. S. Pirozhkov, and N. N. Rosanov, Phys.-Usp. 56, 429 (2013).

${ }^{9}$ T. Z. Esirkepov, S. Bulanov, M. Kando, A. Pirozhkov, and A. Zhidkov, Phys. Rev. Lett. 103, 025002 (2009).

${ }^{10}$ J. Meyer-ter-Vehn and H. C. Wu, Eur. Phys. J. D 55, 433 (2009).

${ }^{11}$ H.-C. Wu, J. Meyer-ter-Vehn, J. Fernandez, and B. Hegelich, Phys. Rev. Lett. 104, 234801 (2010).

${ }^{12}$ D. Kiefer, M. Yeung, T. Dzelzainis, P. Foster, S. Rykovanov, C. L. Lewis, R. Marjoribanks, H. Ruhl, D. Habs, J. Schreiber et al., Nat. Commun. 4, 1763 (2013)

${ }^{13}$ S. V. Bulanov, I. N. Inovenkov, V. I. Kirsanov, N. M. Naumova, and A. S. Sakharov, Kratk. Soobshch. Fiz. 6, 9 (1991).

${ }^{14}$ C. Decker, W. Mori, K. C. Tzeng, and T. Katsouleas, Phys. Plasmas 3, 2047 (1996).

${ }^{15}$ S. V. Bulanov, T. Esirkepov, and T. Tajima, Phys. Rev. Lett. 91, 085001 (2003).

${ }^{16}$ T. Tajima and J. Dawson, Phys. Rev. Lett. 43, 267 (1979).

${ }^{17}$ M. Kando, Y. Fukuda, A. Pirozhkov, J. Ma, I. Daito, L. M. Chen, T. Z. Esirkepov, K. Ogura, T. Homma, Y. Hayashi et al., Phys. Rev. Lett. 99, 135001 (2007).

${ }^{18}$ M. Kando, A. Pirozhkov, K. Kawase, T. Z. Esirkepov, Y. Fukuda, H. Kiriyama, H. Okada, I. Daito, T. Kameshima, Y. Hayashi et al., Phys. Rev. Lett. 103, 235003 (2009).

${ }^{19}$ A. Pirozhkov, J. Ma, M. Kando, T. Z. Esirkepov, Y. Fukuda, L. M. Chen, I. Daito, K. Ogura, T. Homma, Y. Hayashi et al., Phys. Plasmas (1994present) 14, 123106 (2007).

${ }^{20}$ C. Benedetti, C. Schroeder, E. Esarey, F. Rossi, and W. Leemans, Phys. Plasmas 20, 103108 (2013).

${ }^{21}$ N. H. Matlis, S. Reed, S. S. Bulanov, V. Chvykov, G. Kalintchenko, T. Matsuoka, P. Rousseau, V. Yanovsky, A. Maksimchuk, S. Kalmykov et al., Nat. Phys. 2, 749 (2006).

${ }^{22}$ F. Y. Li, Z. M. Sheng, M. Chen, L. L. Yu, J. Meyer-ter-Vehn, W. B. Mori, and J. Zhang, "Radially polarized, half-cycle, attosecond pulses from laser wakefields through coherent synchrotron radiation," preprint arXiv:1401.2799 (2014).

${ }^{23}$ A. Solodov, V. Malkin, and N. Fisch, Phys. Plasmas 13, 093102 (2006).

${ }^{24}$ A. Pukhov and J. Meyer-ter-Vehn, Appl. Phys. B 74, 355 (2002).

${ }^{25}$ W. Lu, M. Tzoufras, C. Joshi, F. Tsung, W. Mori, J. Vieira, R. Fonseca, and L. Silva, Phys. Rev. ST Accel. Beams 10, 061301 (2007).

${ }^{26}$ E. Esarey, C. Schroeder, and W. Leemans, Rev. Mod. Phys. 81, 1229 (2009).

${ }^{27}$ H. Schwoerer, B. Liesfeld, H.-P. Schlenvoigt, K. U. Amthor, and R. Sauerbrey, Phys. Rev. Lett. 96, 014802 (2006).

${ }^{28}$ K. T. Phuoc, S. Corde, C. Thaury, V. Malka, A. Tafzi, J. Goddet, R. Shah, S. Sebban, and A. Rousse, Nat. Photonics 6, 308 (2012).

${ }^{29}$ T. Xu, M. Chen, F. Y. Li, L. L. Yu, Z. M. Sheng, and J. Zhang, Appl. Phys. Lett. 104, 013903 (2014).

${ }^{30}$ F. Y. Li, Z. M. Sheng, Y. Liu, J. Meyer-ter-Vehn, W. B. Mori, W. Lu, and J. Zhang, Phys. Rev. Lett. 110, 135002 (2013).

${ }^{31}$ J. Mu, F. Y. Li, M. Zeng, M. Chen, Z.-M. Sheng, and J. Zhang, Appl. Phys. Lett. 103, 261114 (2013). 
${ }^{32}$ H. T. Kim, K. H. Pae, H. J. Cha, I. J. Kim, T. J. Yu, J. H. Sung, S. K. Lee, T. M. Jeong, and J. Lee, Phys. Rev. Lett. 111, 165002 (2013).

${ }^{33}$ X. Wang, R. Zgadzaj, N. Fazel, Z. Li, S. Yi, X. Zhang, W. Henderson, Y. Y. Chang, R. Korzekwa, H. E. Tsai et al., Nat. Commun. 4, 1988 (2013).

${ }^{34}$ R. Fonseca, L. Silva, F. Tsung, V. Decyk, W. Lu, C. Ren, W. Mori, S. Deng, S. Lee, T. Katsouleas et al., Lect. Notes Comput. Sci. 2331, 342 (2002).
${ }^{35}$ S. Bulanov, M. Yamagiwa, T. Esirkepov, J. Koga, M. Kando, Y. Ueshima, K. Saito, and D. Wakabayashi, Phys. Plasmas 12, 073103 (2005).

${ }^{36}$ H. C. Wu, J. Meyer-ter-Vehn, B. Hegelich, and J. Fernandez, Phys. Rev. ST Accel. Beams 14, 070702 (2011).

${ }^{37}$ A. V. Panchenko, T. Zh. Esirkepov, A. S. Pirozhkhov, M. Kando, F. F. Kamenets, and S. V. Bulanov, Phys. Rev. E 78, 056402 (2008).

${ }^{38}$ H. C. Wu and J. Meyer-ter-Vehn, Eur. Phys. J. D 55, 443 (2009). 